\title{
Traumatic Multi-Finger Amputations After Trying to Rein in a Horse
}

\author{
Rohan Mangal ${ }^{1}$, Tej G. Stead ${ }^{2}$, Latha Ganti ${ }^{3}$, Travis Jasper $^{4}$, Bryan C. Sleigh ${ }^{5}$ \\ 1. Emergency Medicine, John Hopkins University, Baltimore, USA 2. Emergency Medicine, Brown University, \\ Providence, USA 3. Emergency Medicine, Envision Physician Services, Orlando, USA 4. Emergency Medicine, Wellstar \\ Atlanta Medical Center, East Point, USA 5. Emergency Medicine, Mercer University School of Medicine, Macon, USA
}

Corresponding author: Rohan Mangal, rmangal2@jhu.edu

\begin{abstract}
The authors present a case of traumatic, multiple partial-digit amputations caused by the patient entangling three of his fingers in the reins of a moving horse. The patient experienced amputation in fingers III-V on the dominant, right hand. The patient was stabilized and provided analgesia before referral to a surgical center for potential replantation. The emergency management of traumatic finger amputations is reviewed.
\end{abstract}

Categories: Emergency Medicine, Transplantation, Trauma Keywords: digit amputation

\section{Introduction}

Traumatic amputations represent approximately $1 \%$ of all trauma-related injuries. Among the said cases, multiple digit amputations are far less common [1]. In the management of finger amputation, the foremost goal is to restore function in severed parts. Patients with partial-hand amputation experience reduced grip strength and functionality, exacerbated by when fingers III-V are removed [2].

The goals of treating a fingertip amputation are to (1) preserve functional length, (2) achieve durable coverage, (3) achieve near-normal sensibility, (4) prevent adjacent joint contracture, (5) achieve early functional recovery, and (6) provide adequate pain control [3-4].

Replantation can provide a tremendous quality-of-life difference in patients psychologically and functionally. The success of a digit replantation is often contingent on how well-preserved the extremity is. It is well-supported that digit replantation is optimistic within 24 hours of cold ischemia time and 12 hours of warm ischemia time [5]. However, freezing the amputated extremity in water is not recommended [6].

Received 06/17/2019

Review began 06/22/2019 Review ended 06/28/2019 Published 07/18/2019

๑) Copyright 2019 Mangal et al. This is an open access article distributed under the terms of the Creative Commons Attribution License CC-BY 3.0., which permits unrestricted use, distribution, and reproduction in any medium, provided the original author and source are credited.

\section{Case Presentation}

A 25-year-old healthy male, who is right hand dominant, presented to our freestanding emergency department (ED) with right, third, fourth, and fifth finger trauma. The 3rd and 4th digits sustained partial amputations. The 5th digit sustained a complete fingertip amputation distal to the distal interphalangeal joint. The patient's case was the result of helping his friend with a pet horse. The injury occurred from entangling fingers III-V in the reins of the horse before it began galloping away. The degree of bleeding was moderate and the patient reported moderate pain from the incident. There were neither exacerbating factors nor relieving factors. Aside from pain, there were no other related symptoms. Past medical and surgical history was negative; he had no drug allergies and denied any toxic habits.

The patient arrived with a makeshift dressing of his hand and holding the severed distal fingertip in his shirt pocket. The dressing was removed and the hand examined. He had an intact radial pulse, and the hand was overall warm and well perfused. He was unable to fully straighten his middle three fingers at the proximal interphalangeal joints. The exposed tendon was visible in digit IV (Figure 1). The tip of digit III was twisted $180^{\circ}$ such that the nail was visible on the palmar rather than the dorsal aspect of the hand (Figures 2,3 ). 


\section{Cureus}

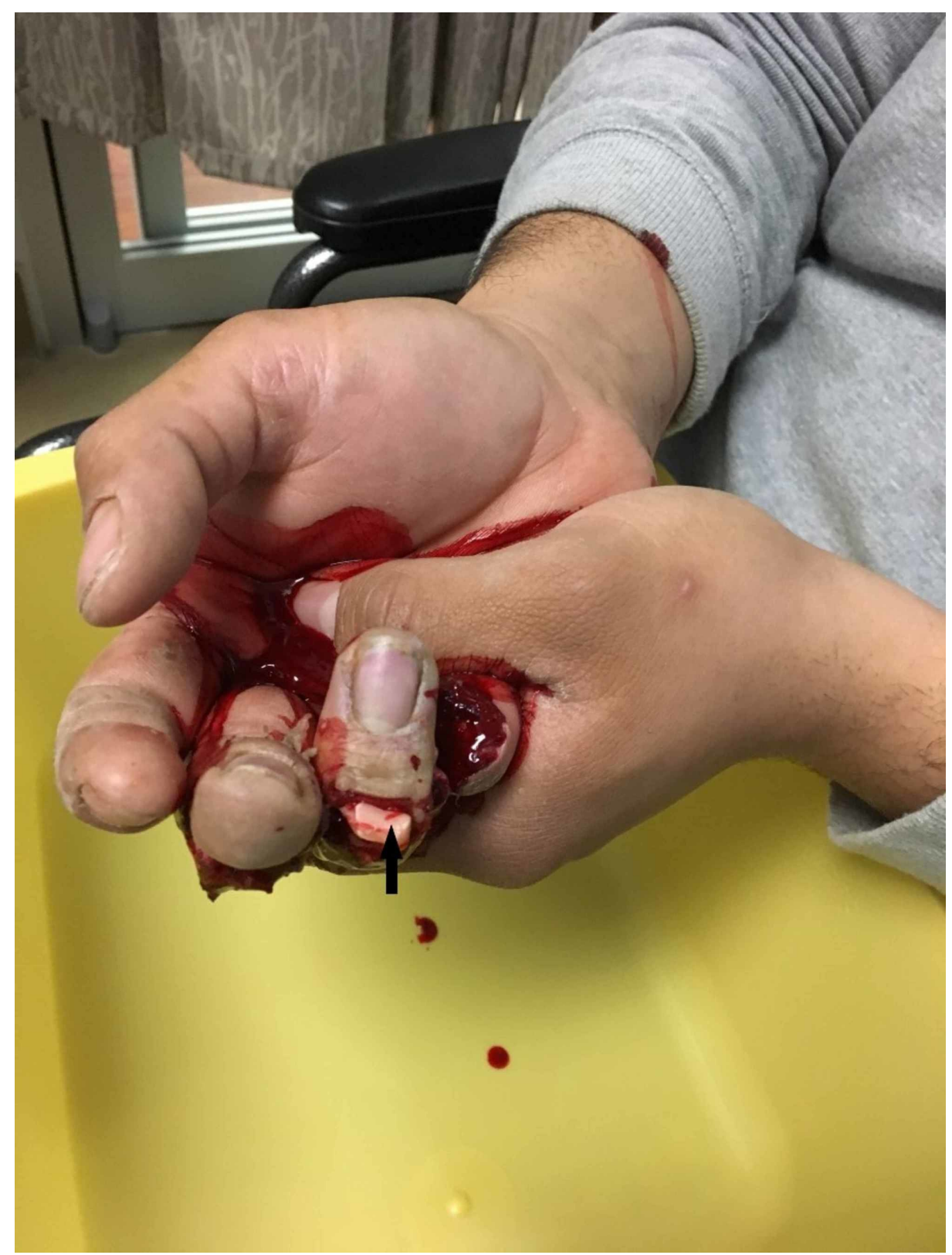

FIGURE 1: Aerial view of multiple digit amputation

Arrow denotes severed tendon. 


\section{Cureus}

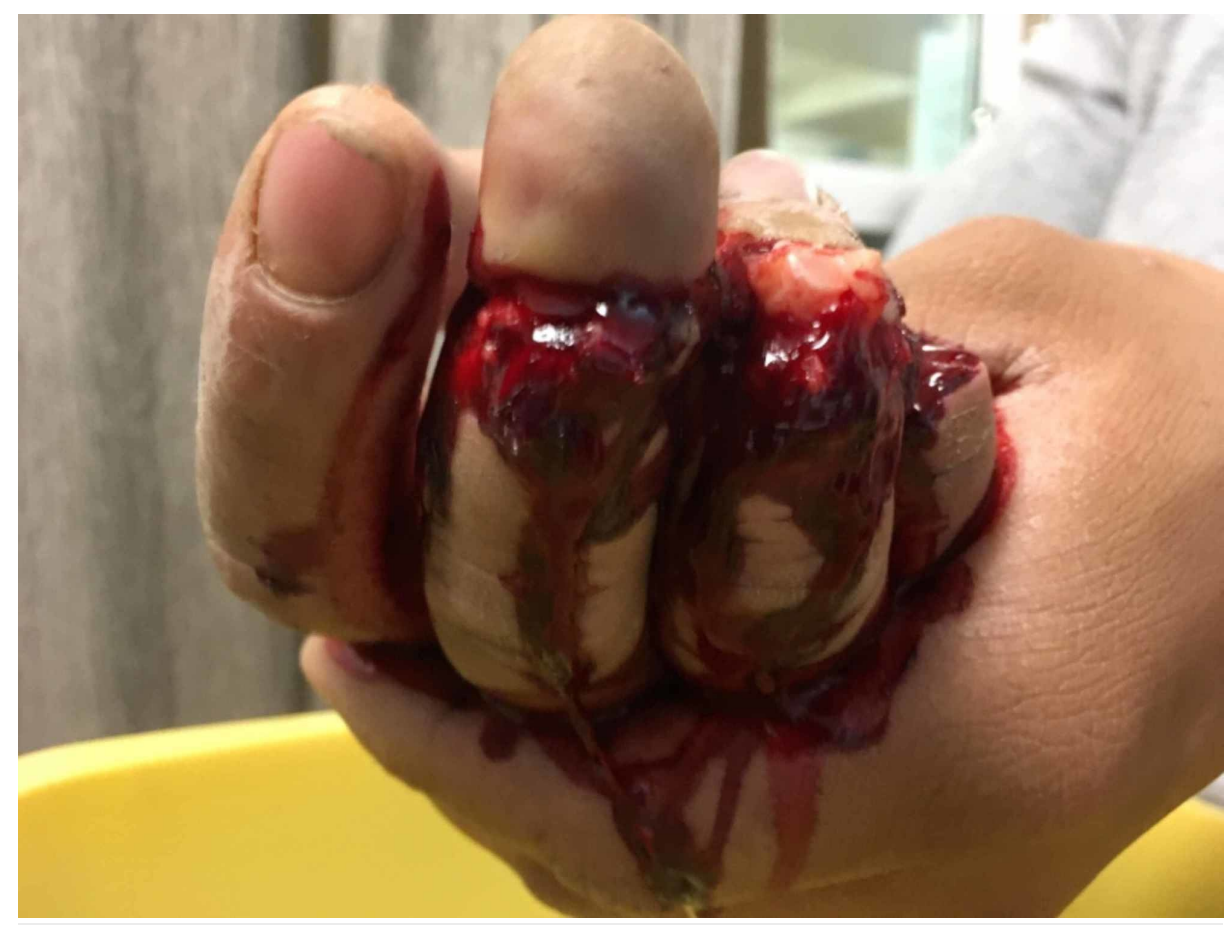

FIGURE 2: Rear view of multiple digit amputation

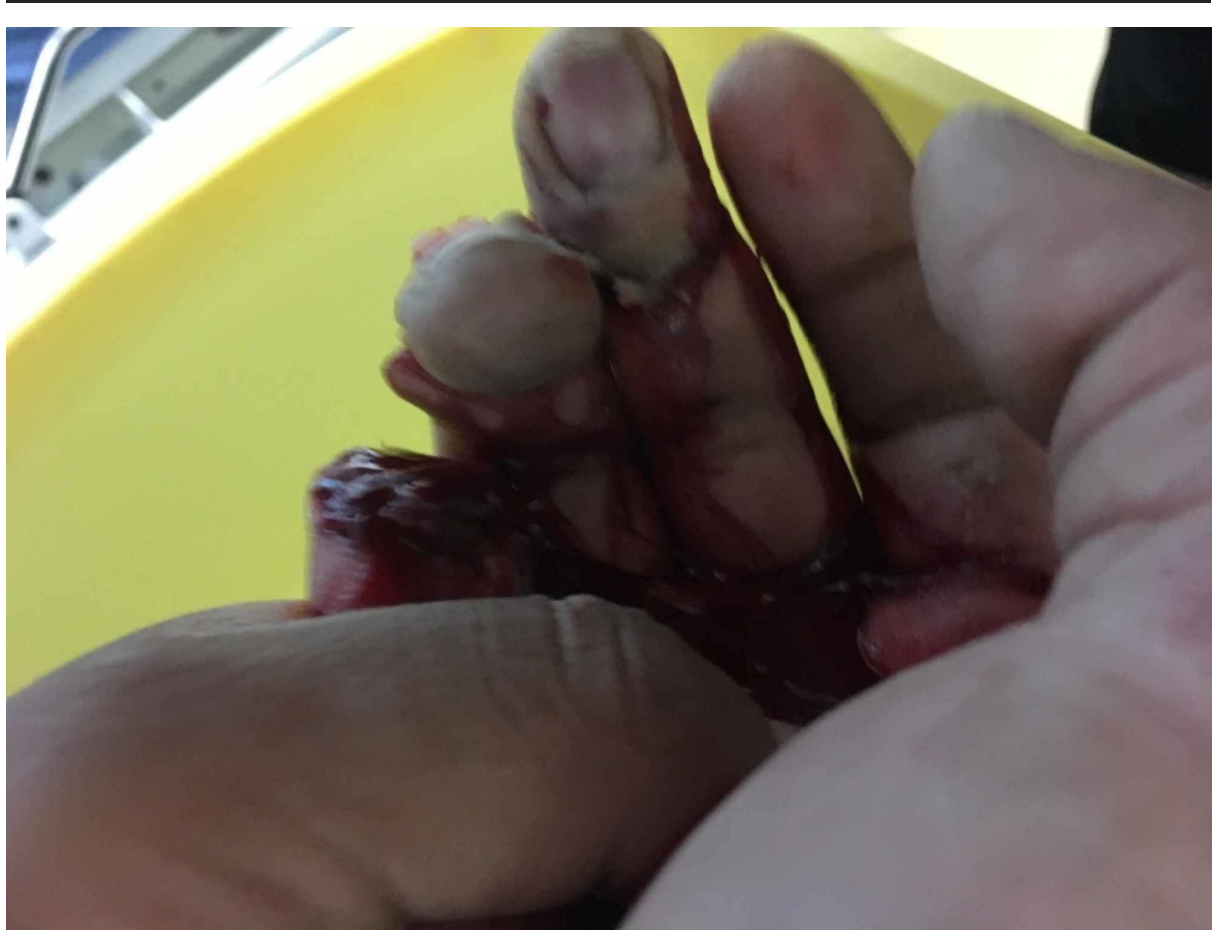

FIGURE 3: View of multiple digit amputation from palm of hand

The patient's last tetanus was at age 15 , and so he was given tetanus toxoid. For analgesia, he received $4 \mathrm{mg}$ of morphine intravenously (IV) and a digital block of fingers III-IV was performed using $1 \%$ lidocaine without epinephrine. One gram of cefazolin IV was given as antibiotic prophylaxis. The severed part was wrapped in saline-moistened gauze and placed in a clean re-sealable plastic bag.

A call was then made to the local trauma center, where he was accepted by the hand surgeon on call. On follow-up, we learned that the patient's fourth fingertip was successfully replanted. 


\section{Discussion}

The emergency management of traumatic finger amputation consists of stabilizing the patient, preserving the severed part if available, and prompt referral to a hand specialist. The best medium for a severed finger is to wrap it in saline-moistened gauze [1,3]. Placing the part directly on ice is not recommended. The optimal analgesia for hand injuries is to provide a digital block. The traditional teaching is that epinephrine should not be used in fingers due to the fear that it could result in finger necrosis or gangrene. However, several comprehensive literature reviews have debunked this myth [7-8].

Patients who incur a multiple digit amputation may experience a significant decrease in hand functioning and grip strength. This injury poses great psychological and social burden and can cause a marked drop-off in quality-of-life (QOL) in both males and females [6].

Replantation is the process of reattaching severed body parts to restore function. Indications for digit replantation include severance of thumb or multiple fingers, as well as amputation at or proximal to the palm. Digit replantation is also considered with a finger amputation at any level in pediatric patients [9].

\section{Conclusions}

When working with potentially dangerous animals, it is important to take proper safety precautions. Amputation of fingers III-V can profoundly lower patients' quality-of-life and demand immediate attention. Replantation should be considered after assessing patient eligibility through indications to restore function in severed body parts. To ensure the success of such a surgical intervention, amputated parts should be preserved in cold saline to maximize ischemic tolerance times.

\section{Additional Information \\ Disclosures}

Human subjects: Consent was obtained by all participants in this study. Conflicts of interest: In compliance with the ICMJE uniform disclosure form, all authors declare the following: Payment/services info: All authors have declared that no financial support was received from any organization for the submitted work. Financial relationships: All authors have declared that they have no financial relationships at present or within the previous three years with any organizations that might have an interest in the submitted work. Other relationships: All authors have declared that there are no other relationships or activities that could appear to have influenced the submitted work.

\section{Acknowledgements}

This research was supported (in whole or in part) by HCA Healthcare and/or an HCA Healthcare affiliated entity. The views expressed in this publication represent those of the author(s) and do not necessarily represent the official views of HCA Healthcare or any of its affiliated entities.

\section{References}

1. Zhang L, Buckley CJ: Digit amputation. StatPearls. 2019, https://www.ncbi.nlm.nih.gov/books/NBK538153/.

2. Kuret Z, Burger H, Vidmar G: Influence of finger amputation on grip strength and objectively measured hand function: a descriptive cross-sectional study. Int J Rehabil Res. 2015, 10.1097/MRR.0000000000000110

3. The nuts \& bolts of finger amputation . (2015). Accessed: June 15, 2019: https://epmonthly.com/article/thenuts-a-bolts-of-finger-amputation/.

4. Digital amputations. (2018). Accessed: June 15, 2019: https://musculoskeletalkey.com/digital-amputations/.

5. Salah MM, Khalid KN: Replantation of multiple digits and hand amputations: four case reports . Cases J. 2008, 10.1186/1757-1626-1-266

6. Mohammed SA, Shebl AM: Quality of life among Egyptian patients with upper and lower limb amputation: sex differences. Adv Med. 2014, 10.1155/2014/674323

7. Illicki J: Safety of epinephrine in digital nerve blocks: a literature review . J Emerg Med. 2015, 10.1016/j.jemermed.2015.05.038

8. Denkler K: A comprehensive review of epinephrine in the finger: to do or not to do . Plast Reconstr Surg. 2001 ,

9. Wolfe VM, Wang AA: Replantation of the upper extremity: current concepts . J Am Acad Orthop Surg. 2015, 10.5435/JAAOS-D-14-00039 